\title{
Expression and localization of nerve growth factor (NGF) in the testis of alpaca (llama pacos)
}

\author{
Haidong Wang¹, Yanjun Dong ${ }^{1}$, Wenhua Chen ${ }^{1,}$, Junping Hei ${ }^{1}$, Changsheng Dong ${ }^{1}$ \\ ${ }^{1}$ College of Animal Science and Veterinary Medicine, Shanxi Agricultural University, China \\ ${ }^{2}$ Hanzhong People's Hospital, China
}

\begin{abstract}
During alpaca testis development and spermatogenesis, nerve growth factor (NGF) may play an important role. The main aim of this study was to determine the expression and localization of NGF in the alpaca testis, and to discuss the important role of NGF in alpaca reproductive characteristics. Immunohistochemical staining technique and real-time PCR were used. The expression of NGF in the same cells one-month old (newborn) alpacas 12-month, and 24-month old alpacas showed significant differences ( $<<0.05)$; 12 - and 24-month old alpacas showed no significant differences ( $p>0.05)$; NGF at different cell stages showed no significant differences $(p>0.05)$. It suggests that NGF may be involved in the regulation of spermatogenesis, which provides direct evidence for NGF action in the alpaca testis during postnatal development and spermatogenesis. (Folia Histochemica et Cytobiologica 2011; Vol. 49, No. 1, pp. 55-61)
\end{abstract}

Key words: alpaca, testis, NGF (nerve growth factor), SABC

\section{Introduction}

The popularity of the alpaca as an agricultural and companion animal has been increasing. There are estimated to be more than 3 million alpaca worldwide $[1,2]$. It is used especially in its native South America in fiber and meat production and packing [3]. But, there are some distinct characteristics of alpaca reproduction. Postnatal development and function of the testes is controlled by a complex interaction of circulating gonadotropins with cytokines and growth factors [4, 5]. The evidence supports a potential regulatory role for nerve growth factor (NGF) in controlling testicular function in mice, rats and livestock species [6]. The concentration of NGF in males is about ten times the concentration in females [2]. The main tissue source of NGF is the submandibular glands and prostate [7-9].

Nerve growth factor and brain-derived neurotrophic factor (BNGF), neurotrophin 3 (NT-3), neu-

Correspondence address: C. Dong,

College of Animal Science and Veterinary Medicine,

Shanxi Agricultural University, Taigu, 030801, Shanxi, China;

tel.: (+ 86 354) 62886 17; fax: (+ 86 354) 62882 08;

e-mail: dongchangsheng2010@hotmail.com rotrophin 4/5 (NT-4/5), and neurotrophin 6 (NT-6) all belong to neurotrophic factor categories [2]. NGF, with a molecular weight of $140 \mathrm{KD}$, isolated from the mouse submandibular gland, is the classic glycoprotein. There are three kinds of peptide chains, $\alpha, \beta$ and $\gamma$, composed of proportions of $\alpha_{2} \beta \gamma_{2}$ peptide chains held together by covalent bonding, where $\beta$ subunit is the active area. There are two types of NGF receptors. One is a low-affinity receptor (LNGFR), with a dissociation constant of $2.3 \times 10^{-11} \mathrm{M}$, molecular weight of $42 \mathrm{kD}$, glycosylated $75 \mathrm{kD}$. It is also called P75 protein, which contains about 400 amino acid residues. The other is a high-affinity receptor (FNGFR), with a dissociation constant of $1.7 \times 10^{-9} \mathrm{M}$, which uses protooncogene Trk to encode the receptor proteins, including three types: TrkA, TrkB, TrkC. Moreover. TrkB, TrkC are not the function receptors [10].

NGF and NGFR have been localized specifically in a variety of animal and human testes, epididymis, prostate and other organs, with NGFR and NGFR mRNA expression $[8,11]$. Knowledge of the regulation of male reproductive (testicular) function in South American camelids such as the alpaca, which exhibit a highly variable and late onset of puberty, is limited [12]. To the best of our knowledge, the intratesticular localization of growth factors and growth 
factor receptors, such as EGF and EGFR, which may modulate testicular development function in the alpaca, has not been examined previously. So, the purpose of the present study is to determine the quantity and cell-specific localization of NGF at three specific stages of the post-natal development of alpaca testes.

\section{Material and methods}

\section{Animals and tissue preparation}

The housing and care of animals and collection of testis samples for use in the described experiments were conducted in accordance with the International Guiding Principles for Biomedical Research Involving Animals (http://www.cioms.ch/publications/guidelines/1985_texts_of_guidelines.htm). Testis biopsies were obtained from 12 male alpacas (four of them one month old, four 12 months old and four 24 months old) which were the subjects of the study. Every effort was made to minimize both the suffering and the number of animals used.

\section{Immunohistochemistry}

The optimal conditions for the immunohistological application of our biological material were as previously presented $[12,13]$. The kit used was the StreptAvidin-Biotin-enzyme Complex, SABC System, Peroxidase DAKO (Biomeda Staining Kit): an indirect immuno-histochemistry method using streptavidin-biotin complex that allows the amplification of marking. Free-floating sections of alpaca testis were washed three times, for three minutes each, in 0.1M PBS and were then incubated at room temperature in $3 \%$ hydrogen peroxide for 15 minutes to block the action of any endogenous peroxidase. After washing with $0.1 \mathrm{M}$ PBS three times for 15 minutes, there was a 10-min boiling in $\mathrm{H}_{2} \mathrm{O}$ containing $0.01 \mathrm{M}$ citric acid. This was followed by a 20-min immersion in PBS containing $5 \%$ BSA (bull serum albumin) at $37^{\circ} \mathrm{C}$. Afterwards, sections were incubated for $15-24$ hours at $4^{\circ} \mathrm{C}$ in one of the primary antibody solutions (polyclonal neurotrophin antisera for NGF, diluted 1:75), and for 30 minutes at room temperature. Following three washes in 0.1M PBS for five minutes each, sections were incubated with secondary antibodies (biotinylated anti-rabbit IgG, 1:100 dilution; boster for 20-30 minutes at $37^{\circ} \mathrm{C}$. After washing with $0.1 \mathrm{M}$ PBS three times for 15 minutes, sections were incubated with an avidin-biotin-peroxidase reagent (1:200 dilution, SABC Elite; boster), and the immunoreaction products were visualized by placing the sections in a staining solution containing $0.04 \% \mathrm{DAB}$ (3,3'-diaminobenzidine),
$0.06 \%$ nickel sulfate, and $0.06 \%$ hydrogen peroxide for 5-10 minutes. Nuclei were counterstained with hematoxylin. Then they were mounted, dehydrated, coverslipped, and observed under a light microscope (NIKON).

In negative controls, PBS was substituted for the primary antibody in the first reaction [14].

\section{Optical density of NGF collection}

Forty immunohistological sections were made at every stage. Using an image analysis system, four positive NGF data were collected from the same cell for each section, and these data were used as statistics.

\section{Total RNA isolation}

Total RNA was extracted with Trizol Reagent (invitrogen) according to the manufacturer's instructions, and the integrity of the total RNA was confirmed using a $1 \%$ agarose gel electrophoresis.

\section{Real-time PCR for NGF}

Real-time PCR was employed to obtain quantitative data on the differences between NGF mRNA expression throughout the testis developmental cycle in alpacas. Briefly, the total RNA was polyadenylated using poly A polymerase and ATP. Complementary DNA was generated using the NCode universal reverse primer, and real-time PCR was performed using two sequence-specific primers for NGF (forward primer, 5'GCAGTGAGGTGCATAGCGTAA3', Reverse Primer, 5'AGTGGGCTTCAGGGACAGAG 3'); and $\beta$-actin (Forward Primer, 5'CATCCGTAAAGA CCTCTATGCCAAC 3', Reverse Primer, 5' ATGGAGCCACCGATCCACA3'). The $20 \mu \mathrm{L}$ PCR reaction included $10 \mu \mathrm{L}$ SYBR Premix Ex Taq, $1 \mu \mathrm{L}$ PCR Forward Primer $(10 \mu \mathrm{mol} / \mathrm{L}), 0.8 \mu \mathrm{L}$ PCR Reverse Primer $(10 \mu \mathrm{mol} / \mathrm{L}), 0.4 \mu \mathrm{L}$ ROX Reference Dye II (50 $\times), 2.0 \mu \mathrm{L}$ tample cDNA, $6 \mu \mathrm{L}$ RNase Free $\mathrm{d}_{2} \mathrm{O}$. The reactions were incubated in a 96 -well plate at $95^{\circ} \mathrm{C}$ for two minutes, followed by 40 cycles at $95^{\circ} \mathrm{C}$ for five seconds and $60^{\circ} \mathrm{C}$ for 30 seconds; all reactions were performed in triplicate on the Stratagene Mx3005P Real-Time PCR system. The abundance of NGF was normalized relative to that of $\beta$-actin snRNA [15].

\section{Statistical analysis}

All experiments were performed in triplicate. Values were expressed as means \pm SD in the results and calculations. Differences in the abundance of NGF mRNA in testis samples with distinct developmental cycle in alpaca were determined by an analysis of 
variance, and differences in protein abundance for NGF were also determined by an analysis of variance. A p-value of less than 0.05 was considered statistically significant.

\section{Results}

\section{Immunoreactive localization of NGF in alpaca}

Immunohistochemical SABC methods indicated that alpaca NGF protein showed brown in the immunoreactive cells of testes. Product-specific immune response was evenly distributed in the perinuclear cytoplasm. In the one month old alpacas, seminiferous tubules had a small diameter, thin walls, a cavity which was not obvious, many spermatogonia, primary spermatocytes, Sertoli cells and a few stromal cells. Within the seminiferous tubules arranged in groups of spermatogonia disorder, Sertoli cells and spermatogonia boundaries were not obvious. NGF was positive in the Sertoli cells, spermatogonia, and in primary spermatocytes (Figure 1).

In the 12-month old alpacas, the seminiferous tubules had developed, the diameter had increased, but the cavity was not very obvious, the stromal cells had increased and improved to support cell development, and the spermatogonia had progressive differentiation into primary spermatocytes and secondary spermatocytes. There was also a small amount of mature sperm cells. There was the strongest immunoreactive NGF signal for research, mainly in spermatogenic cells, Sertoli cells, interstitial areas that had also started to brown in reaction, and scattered between the two seminiferous tubules, moreover, there was a strong immune response (Figure 1). In the 24-month old alpacas, development of seminiferous tubules had been completed, the lumen was clear, spermatogonia were arranged in multiple layers, and showed a large number of mature sperm cells. NGF positive was mainly found in stromal cells, Sertoli cells and germ cells, but the immune response intensity had decreased compared to the 12-month olds. NGF was negative in the control experiment sections (Figure 1).

Use of an image analysis system picked up the optical density of NGF positive tissue at three specific stages of alpaca testes, and obtained an average optical density by statistical analysis (Figure 2).

\section{Real-time PCR}

The results of real-time quantitative PCR analysis showed that the expression of the NGF gene was detected at the mRNA level in three specific stages of the post-natal development of the alpaca testes. How- ever, it was found that, compared to 1-month olds, 12-month old and 24-month old alpacas' NGF mRNA expression had increased 1.816-fold and 0.565-fold, respectively (Figure 3). This indicated that NGF reached a peak at the sexual maturity of the alpaca, but NGF expression had begun to rise before that, while the expression began to decline in late sexual maturity, and to maintain a certain level. Differentiation of testis and sperm had already matured.

\section{Discussion}

Olson et al. [16] have demonstrated that the NGF immune complex exists in the spermatogenic cells at all levels. Subsequent research has indicated that NGF exists in the spermatogenic cells at all ages, in mature spermatozoon and the seminiferous tubules of rats and mice. Furthermore, NGF participates in the adjustment of testosterone. NGF plays an important role in promoting the formation and development of the testis and the differentiation, maturation and movement of the spermatozoon $[17,18]$. This review reveals that immunoreactive material NGF is expressed in all Leydig cells, peritubular myoid cells, Sertoli cells and spermatogenic cells of 1-month old, 12-month old and 24-month old alpaca testes. This result supports work previously carried out.

Leydig cells secrete androgens. Androgens can promote the formation of spermatozoon, the growth and the differentiation of male reproductive organs, and maintain secondary sexual characteristics and sexual function [19]. Immunohistochemical staining and real-time PCR showed that NGF has strong expression in the Leydig cells of the 1-month old, 12-month old and 24-month old alpaca testis. Specifically, there is a significant difference in the expressional quantity between them.

The research by Wrobel et al. [20] into cattle testis showed that NGF in an immunohistochemistry experiment on the testis-derived mesenchymal cells of 7-month old fetal cattle was strongly positive. However, the result became negative when testis-derived mesenchymal cells differentiated into interstitial cells and peritubular myoid cells. This research indicates that NGF plays an important role in adjusting the differentiation and maturation of interstitial cells.

Koeva et al. [21] found the expression of p75 and TrkA in human Leydig cells. These results showed that Leydig cells, peritubular myoid cells and Sertoli cells were some of the potential origins of testicular NGF. NGF and its receptor played a certain role in adjusting the synthesis of steroid hormone in Leydig cells through autocrine and paracrine function. It was postulated that the adjustment of the activity of the steroid gene affects the function of the Leydig cell. 


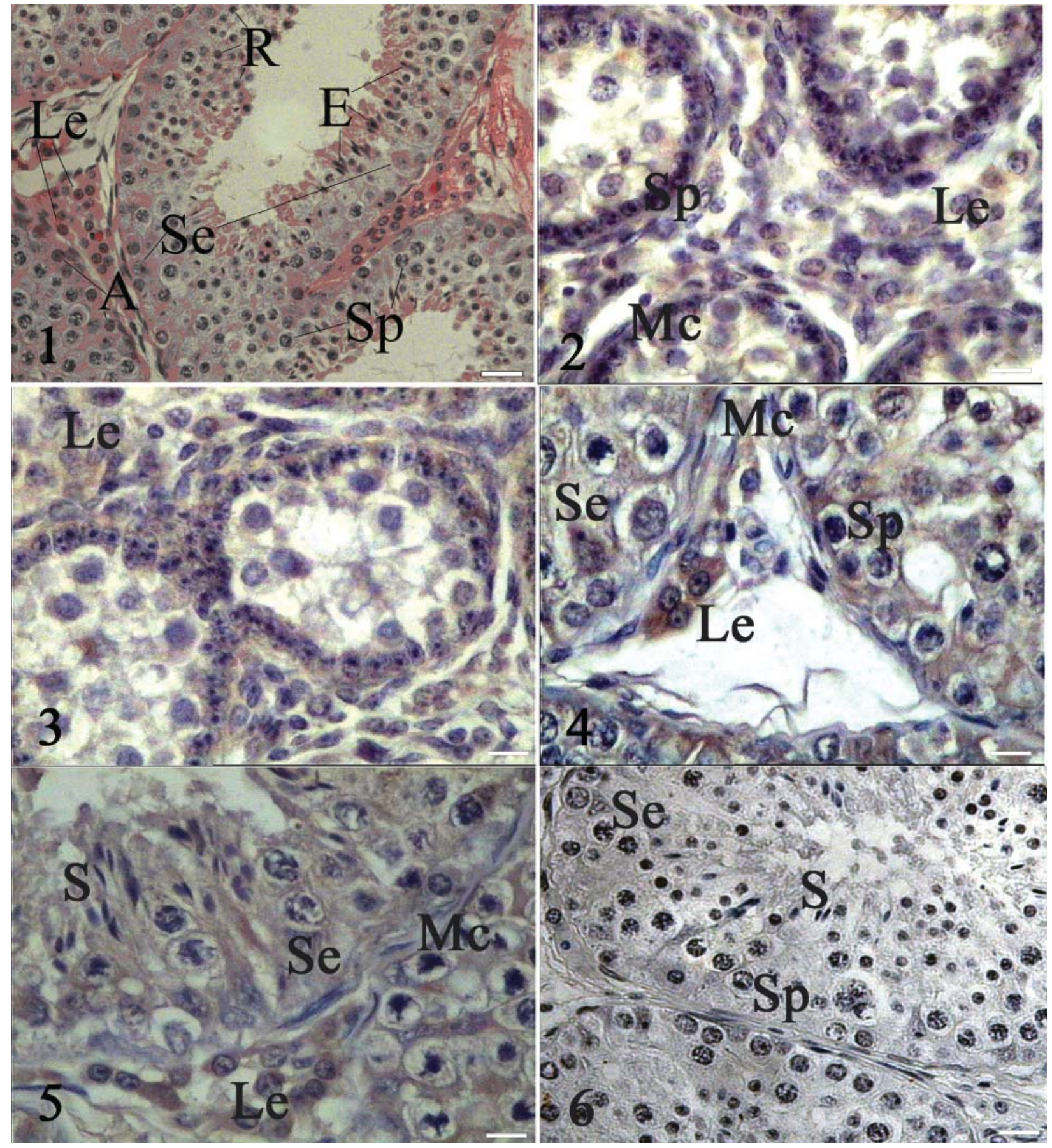

Figure 1. Immunohistochemical SABC method, hematoxylin counterstain $(\times 40)$. Le — Leydig cells; Se — Sertoli cells; Sp — spermatocytes; A — A type spermatogonia; R — round spermatids; E — elongating spermatids; S — spermatids; bar $=50 \mu \mathrm{m}$. 1. The histological structure of alpaca testis (HE). 2. Positive expression of NGF in testis of 1-month old alpacas, with brown staining in spermatogonia cell, Sertoli cell, somatic cell and Leydig cell. 3. Positive expression of NGF in testis of 12-month old alpacas, with brown staining in spermatogonia cell, Sertoli cell, somatic cell, myoid cell and Leydig cell. 4, 5. Positive expression of NGF in testis of 24-month old alpacas, with brown staining in spermatogonia cell, Sertoli cell, somatic cell, spermatid and Leydig cell. 6. NGF was negative in the control experiments sections

NGF not only has an influence on the growth of the testis and the function of the interstitial cell, but is also closely related to the formation of spermatozoon. NGF immune response material exists in alpaca spermatogenic cells at all levels. Cao et al. [22] found NGF mRNA expressed in the cytoplasm of the spermatogenic cells at all levels. The positive degree of the experiment was gradually enhanced from spermatogonia to spermatozoon. Moreover, for spermatozoon, the more mature they became, the more posi- 


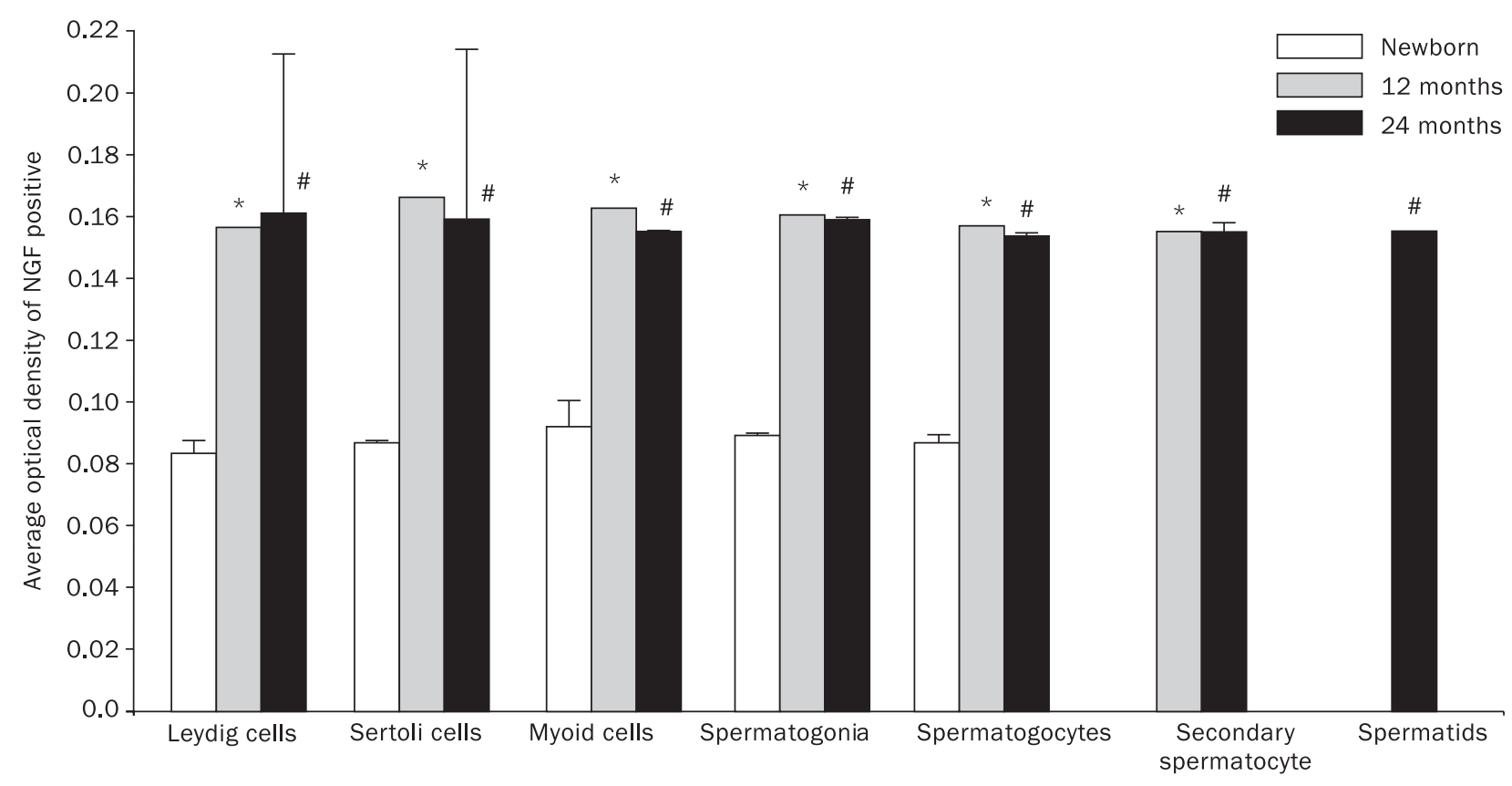

Figure 2. Average optical density of NGF positive tissue in alpaca testis; * $p<0.05$, 1-month old alpacas vs. 12-month old alpacas; ${ }^{\#} \mathrm{p}<0.05$, 1-month old alpacas vs. 24-month old alpacas and; 12- and 24-month old alpacas showed no significant differences ( $\mathrm{p}>0.05)$; NGF at different cell stages showed no significant differences $(\mathrm{p}>0.05)$

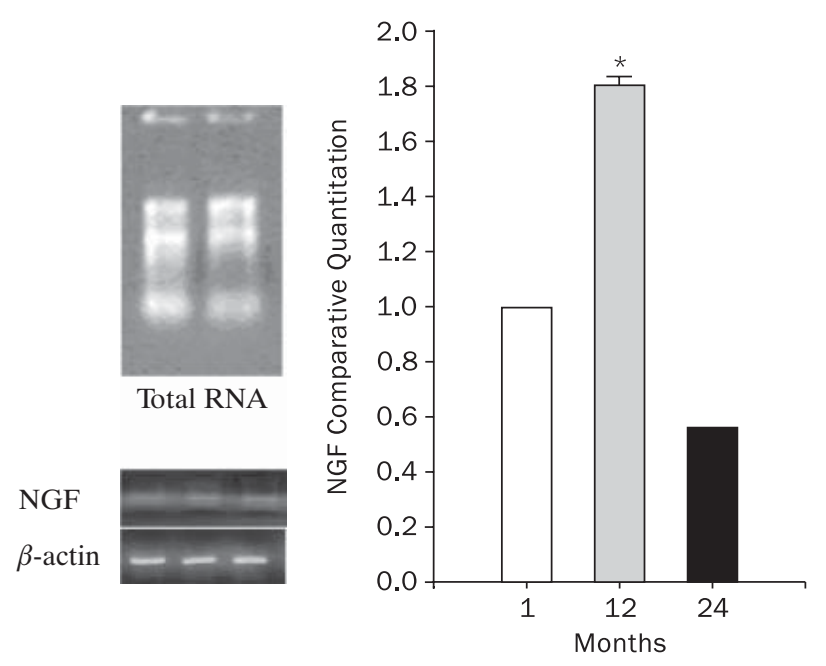

Figure 3. Level of NGF mRNA in different age group mouse testis; *p < 0.05, 1-month old alpacas vs. 12-month old alpacas; $\# \mathrm{p}<0.05,12$-month old alpacas vs. 24-month old alpacas

tive. This indicates that constant participation of NGF is essential to the formation of spermatozoon. However, the NGF mRNA of spermatozoon tails was strongly positive, which may be related to the activity and the mobility of spermatozoon.

Parvinen et al. [23] found that the synthesis of spermatogenic cell DNA during the mature division stage was gradually enhanced when the dose of NGF was increased through the culture of rat seminiferous tubules. NGF can maintain the thickness of the semini- ferous epithelium of testis, prevent the degeneration of spermatogenic cells, and also promote the growth and the maturation of spermatogenic cells. The testis seminiferous epithelium includes Sertoli cells and spermatogenic cells at all levels. The formation and maturation of spermatozoon is regulated by many factors, with androgen being one of the most important.

Our research showed that NGF is expressed in Sertoli cells and spermatogenic cells at all levels. On the one hand, NGF directly affects the formation of spermatozoon through NGF-R. On the other hand, NGF combines with the corresponding receptor of Sertodi cells, which increased the content of the androgen binding protein mRNA in Sertoli cells. Thus, the ability of concatenate male hormone of Sertoli cells was enhanced, prompting the meiosis of spermatogonia and improving the formation and maturation of spermatozoon.

The present study showed no significant difference in the expressional quantity of NGF between different cells of the same age ( $p>0.05)$, which is not identical to the above mentioned research. This is related to the unique reproductive habits of alpaca and the differences between species. Perhaps this could be related to the methods and different anti-bodies used in the experiment. It remains to be further studied. However, it is certain that, like other species, the alpaca spermatogenic cells doesn't merely store NGF it can also synthesize and secrete NGF. NGF, as a meiosis growth 
factor, can promote the development and maturation of spermatogenic cells. It could be inferred that NGF plays an important role in the formation of alpaca spermatozoon.

The experiment which used the real-time PCR was a dynamic analysis of testicular tissue NGF mRNA in different development stages. The results showed that NGF mRNA expression in testicular tissue exists in 1-month old alpacas. Furthermore, the 12-month old alpacas' expressional quantity was much higher than that of the 1-month old alpacas. Male alpaca can reach sexual maturity when they are 12 months old, then they cannot be used for breeding. The expressional quantity of the testicular tissue NGF mRNA of 24-month alpacas will be at a certain level and below the level of previous sexual maturity. Djakiew et al. [24] reported NGF-R (including P75 LNGFR and Trk-R) mRNA expressed at a high level in the testicular tissue of mice aged between ten and 20 days. As the mice get older, the expression begins to decline. The result of this experiment was consistent with the research into the trend in alpacas.

The interstitial cells in the testicular tissue of 1-month old alpacas were reduced and were mainly fibroblast. The development of Sertoli cells was still not perfect. Along with the increase of age, Sertoli cells continue to grow and their functions continue to improve. Thus, secretion gradually increases. An alpaca younger than 12 months old is in its adolescence. During this period, the development of the reproductive system is rapid. It is essential to testicular tissue to secrete plenty of protein and local factors to meet the development needs of the reproductive system.

The local factor produced by testicular tissue may be very important to the regulation of hormone activity. Local factor can be regarded as the substance which can regulate hormone activity and conduct the signal between cells. After 12 months, alpacas reach sexual maturity. The content of the testicular tissue NGF mRNA declines to a lower level, and maintains that level. This phenomenon may be because the tube diameter of the seminiferous epithelium in testicular tissue has reached its peak, and it no longer increases in thickness. The various physiological functions and structures of testicular tissue have already been stabilized. Synthesis and degradation of testicular tissue is balanced. Compared to the prophase of youth, the demand on the synthesis of testicular tissue local factor has decreased.

Our results showed that NGF mRNA of alpaca exists in the development of puberty, in the improvement in function, and in the formation of spermatozoon in the testicular tissue. And with increased age its expressional quantity will be a dynamic trend. In alpaca testicular tissue development, the high expression of NGF mRNA may be related to the development of the testicular tissue itself, the perfection of form and to the decline of its position.

With increased age, the development of testicular tissue, and the maturation and expansion of seminiferous tubules, the mRNA expression of NGF gradually declines. The distribution of testicular tissue NGF mRNA transfers from mesenchyme to germinal cell, but the expression level of adult testicular tissue NGF mRNA still remains at a certain level to participate in the maturation and movement of spermatozoon. This suggests that NGF plays an important biological role in the formation and development of the alpaca's testicular tissue and in the formation and movement of spermatozoon, which will contribute to the further exploration of the reproductive mechanism of the alpaca.

\section{Acknowledgements}

The present research was supported by a grant from the Ministry of Agriculture of the People's Republic of China (No. 2003-Z86). We would also like to thank Ying Wang, the general manager of the Scientific Research Base of Shanxi Agricultural University (Shanxi, China), for provision of the animals used in this study.

\section{References}

1. Lupton CJ, McColl A, Stobart RH. Fiber characteristics of the huacaya alpaca. Small Rumin Res. 2006;64:211-224.

2. Gregor MB. Production attributes and relative value of alpaca fleeces in southern Australia and implications for industry development. Small Rumin Res. 2006;61:93-111.

3. Renieri C. Pigmentation in domestic mammals, with reference to fine fiber producing species. In: Laker JP, Bishop SC (eds). Genetic improvement of fine fiber producing animals No.1. EFFN, Peebles, UK 1995;24:113-136.

4. Skinner MK. Cell-cell interaction in the testis. Endocr Rev. 1991;12:45-77.

5. He JP, Dong CS, You RL et al. Localization of epidermal growth factor (EGF) and its receptor (EGFR) during post-natal testis development in the alpaca (Lama pacos). Anim Reprod Sci. 2009;116:155-161.

6. Zhang S, Chen CH, Zheng ZX. Research of nerve growth factor. Strait Pharmaceutical Journal. 2003;15:8-10.

7. Harer GP, Glanville RW, Thoenen H. The purification of nerve growth factor from bovine seminal plasma.J Biol Chem. 1982;275:8541-8548.

8. Shikata H, Utsumi N, Hiramatsu M et al. Immunohistochemical localization of nerve growth factor and epidermal growth factor in guinea pig prostate gland. Histochemistry 1984;80:411-415.

9. Li CM, Watanabe G et al. Expression of Nerve Growth Factor (NGF), and its receptors TrkA and P75 in the reproductive organs of the adult male rats. Zoological Society of Japan 2005;22:933-937.

10. Rosemhal A, Goeddel DV, Nguyen et al. Primary strcture and biological activity of novel human neurotrophic factor. Neuron 1990;4:767-773. 
11. Levi-Montalcini R. The nerve growth factor thirty-five years Later. Science 1987;237:1154-1162.

12. Tibary A, Vaughan JL. Reproductive physiology and infertility in male South America camelids: a review and clinical observations. Small Ruminant Res. 2006;61: 283-298.

13. Yan YP, Dong CS, Hei JP et al. Expression and localization of transforming growth factor- $\beta 1$ of alpaca testis. Acta Veterinaria and Zootechnica sinica 2008;39:97-102.

14. Ayer-Lelievre C, Olson L, Persson H. Nerve growth factor mRNA and protein in the testis and epididymis of mouse and rat. Proc Natl Acad Sci. 1988;85:2628-2632.

15. Knackstedt MK, Zenclussen AC, Hertwig K et al. Th1 cytokines and the prothrombinase fgl 2 in stress-triggered and inflammatory abortion. Am J Reprod Immunol. 2003;49: 210-220.

16. Olson L, Ayer-Lelievre C, Ebendal T. Nerve growth factorlike immunoreactivities in the testis and epididymis of mouse and rat. Cell Tissue Res. 1987;246:275-286.

17. Lipps BV. Isolation of nerve growth factor (NGF) from human body fluid; saliva, serum and urine: comparison between cobra venom and cobra serum NGF. J Nat Toxins 2000;9: 349-356.
18. MacGrogan D, Despres G, Romand R et al. Expression of the $\beta$-nerve growth factor gene in male sex organs of the mouse, rat and guinea pig. Neurosci Res. 1991;28:567-573.

19. Seidl K, Buchberger A, Erck C. Expression of nerve growth factor and neurotrophinreceptors in testieular cells suggest novel roles for neurotrophins outside the nervous system. Reprod Fertil Dev. 1996;8:1075.

20. Wrobel KH, Biekel D, Schimmel M et al. Immunobistochemical demonstration of nerve growth factor receptor in bovine testis. Cell Tissue Res. 1996;285:189.

21. Koeva Y, Davidof M, Popova L. Immunocytochemical expression of p75 LNGFR and trkA in leyding cells of the human testis. Folia Med (Plovdiv). 1999;41:53.

22. Cao Z, Lu L, Ding F et al. Relation ship between levels of NGF mRNA and maturation and functions of testis spermatogenic cell. Chin J Androl. 1996;10:136-140.

23. Parvinen M, Pelto-Huikko M, Soder O et al. Expression of beta-nerve growth factor and its receptor in rat seminiferous epithelium:specific function at the onset of meiosis. $J$ Cell Biol. 1992;117:629.

24. Djakiew D, Pflug B, Dionne IC et al. Postnatal expression of nerve growth factor receptors in the rat testis. Biol Reprod. 1994;51:214-221. 